\title{
Rheumatology in the current era: the challenge of success
}

\author{
David S Pisetsky
}

Impressive advances in therapy mean that rheumatologists can treat rheumatoid arthritis (RA) and other forms of inflammatory arthritis with remarkable efficacy. As a result of current therapy, fewer patients with RA show deterioration and the incidence of severe disability is diminishing. Indeed, remission in RA-once a dream - is increasingly becoming a reality (Goekoop-Ruiterman YP et al. [2007] Ann Intern Med 146: 406-415). Not too long ago, investigators believed that this type of progress would require drastic or dangerous interventions, such as total lymphoid irradiation (Uhrin Z et al. [2001] Arthritis Rheum 44: 1525-1528) or drainage of the thoracic duct (Paulus HE et al. [1977] Arthritis Rheum 20: 1249-1262); that RA could be managed by pills and injections was completely unforeseeable. Although the unmet need in RA is often discussed as new products are launched, in reality this need is getting smaller. The rate of complications in $\mathrm{RA}$ is decreasing, and patients rarely need hospitalization for this disease.

Given such progress, rheumatology should be thriving as a subspecialty and booming as a bastion of scientific medicine. Instead, in many places in the US, rheumatology seems to be in decline, besieged by diminished status, unrelenting financial pressure and a dwindling supply of academic leaders. The source of these woes is as curious as it is unexpected. The source is success. Indeed, the progress in RA treatment follows a pattern in rheumatology - almost unique to this subspecialty - as a once serious disease is controlled or eliminated at relatively low cost. In the West, rheumatic fever is essentially gone, and gout can be prevented by a biochemical inhibitor. Fortunately, as each of these conditions has all but disappeared, a new, direr one has not

\section{The source of these woes is as curious as it is unexpected. The source is success}

DS Pisetsky is Professor of Medicine and Immunology in the Division of Rheumatology and Immunology at the Duke University Medical Center, Durham, NC, USA.

\section{Competing interests}

The author declared no competing interests.

www.nature.com/clinicalpractice doi:10.1038/ncprheum0791 replaced it. This situation, although fortuitous for the health of mankind, can threaten rheumatology, as the need for its services and its intellectual driving force shrink.

The current baby boom is unlikely to spur the growth of this discipline. There are few medical options available for the treatment of osteoarthritis, and orthopedic surgeons will take care of the most serious joint complaints. In another arena, the treatment of bone disease has become routine following the development of a reliable diagnostic test (dual-energy X-ray absorptiometry) and a burgeoning repertoire of drugs that includes a monthly pill and a yearly infusion. The present is not all bright, however. Systemic lupus erythematosus, scleroderma and vasculitis persist as painful and destructive diseases, but they are too uncommon to propel the expansion of a subspecialty.

If the past can predict the future, outcomes in patients with inflammatory disease will continue to improve. Such improvements are, of course, to be welcomed, but could challenge rheumatology's core and should cause a fundamental rethinking of important issues. How large should rheumatology be? What role should this discipline have in academic medical centers? What constitutes an exciting and engaging research agenda for RA when existing therapy already yields a high frequency of remission?

Success is a wonderful thing, but it can be like winning the lottery. Both come with hidden costs and, as the money flows, weakness can set in. It is time for rheumatologists to acknowledge the potential for weakness. What will it take to make the future of rheumatology as successful as its past? The answer to this question is unknown, but it is clearly time to ask. 